\title{
The Impact of Correlation on Multi-Antenna System Performance: Correlation Matrix Approach
}

\author{
S. Loyka, A. Kouki \\ Department of Electrical Engineering, Ecole de Technologie Superieure \\ 1100, Notre-Dame St. West, Montreal (Quebec), H3C 1K3, Canada \\ Email: sergey.loyka@ieee.org
}

\begin{abstract}
A universal upper bound on the MIMO architecture capacity, which is not limited to a particular scenario, is derived in this paper using the correlation matrix approach and the Jensen's inequality. This bound accounts for both transmit and receive branch correlation in such a way that the impact of these branches can be estimated separately, which simplifies the procedure substantially and also allows to decide which site is responsible for capacity reduction, which is not easy to do using traditional approaches. Further, using the results above and the Salz-Winters model of fading spatial correlation, it is demonstrated that the correlation has no impact on the MIMO capacity provided that the two-element antenna array beamwidth is smaller than the angle spread of the incoming multipath signals. A fundamental tradeoff between MIMO capacity and diversity order is also pointed out.
\end{abstract}

\section{INTRODUCTION}

Multiple-input multiple-output (MIMO) communication architecture, which employs multiple antennas at both the transmitter and the receiver, has recently emerged as a new paradigm of extremely spectrum-efficient wireless communications in rich multipath environment [1]. Suffice it to say that unprecedented wireless spectral efficiencies, ranging from $20-40 \mathrm{bit} / \mathrm{s} / \mathrm{Hz}$, have been demonstrated in a laboratory environment [2], which are simply unattainable using traditional techniques. Even higher spectral efficiencies may be achieved in certain environments when the system design is optimal. However, in real-life conditions the MIMO channel capacity may be limited due to several factors. One of the most important such factors is the correlation between sub-channels of the matrix channel [3-5]. The MIMO capacity achieves its maximum for completely uncorrelated matrix channel. The correlation between individual receive and/or transmit branches results in capacity decrease. Several models have been used to study this phenomenon. Their application is typically limited to some specific scenarios.

In this paper, using Jensen's inequality, we derive the universal upper bound on the MIMO channel capacity, which is not limited to some specific cases. We also demonstrate how to apply the results obtained for diversity combing to the MIMO system analysis using the upper bound above. This upper bound accounts for both transmit and receive branch correlation in such a way that the impact of these branches can be estimated separately, which simplifies the computational procedure substantially and allows to decide which site is responsible for capacity reduction.

We further use the results above and the Salz-Winters model of the fading spatial correlation [7] in order to predict the MIMO capacity in a realistic electromagnetic environment. We study the upper bound on the MIMO capacity as a function of the antenna spacing, the average angle and angular spread of the incoming multipath and demonstrate that the correlation has no impact on the capacity provided that the two-element array beamwidth is smaller than the angle spread of the incoming multipath signals.

The new compound upper bound and simple approximations of the MIMO capacity, derived in this paper, may be used as an efficient design tool because they do not require extensive statistical simulations involving large matrix operations. Thus, efficient optimization procedures are possible.

\section{UPPER BOUND ON MIMO CHANNEL CAPACITY}

For a fixed linear $n \times n$ matrix channel with additive white gaussian noise and when the transmitted signal vector is composed of statistically independent equal power components each with a gaussian distribution, the channel capacity is [1]:

$$
C=\log _{2} \operatorname{det}\left(\mathbf{I}+\frac{\rho}{n} \mathbf{H} \cdot \mathbf{H}^{+}\right) \text {bits } / \mathrm{s} / \mathrm{Hz},
$$

where $n$ is the number of transmit/receive antennas (we consider here the case when the number of transmit and receive antennas are equal), $\rho$ is the signal-to-noise ratio (SNR), $\mathbf{I}$ is $\mathrm{n} \times \mathrm{n}$ identity matrix, $\mathbf{H}$ is the normalized channel matrix, which is considered to be frequency independent over the signal bandwidth, and "'" means transpose conjugate. We adopt here the following normalization condition:

$$
\sum_{i, j=1}^{n}\left|h_{i j}\right|^{2}=n
$$

where $h_{i k}$ denotes the components of $\mathbf{H}\left(h_{i j}\right.$ is the transfer factor between $j^{\text {th }}$ transmit antenna and $i^{\text {th }}$ receive antenna). Hence, $\rho / n$ is the average per-branch SNR, i.e. $\rho$ is the ratio of total received power (in all branches) to the per-branch noise level. Some other kinds of the normalization can also 
be used, but in this case $\rho / n$ will have a slightly different meaning. All the results in this paper hold true for other normalizations as well.

When the channel is random (stochastic), then the capacity is random, too. The mean (ergodic) capacity can be defined in this case as [13]:

$$
\langle C\rangle=\left\langle\log _{2} \operatorname{det}\left[\delta_{i j}+\frac{\rho}{n} \cdot r_{i j}\right]\right\rangle,
$$

where $r_{i j}$ is "instantaneous" correlation matrix,

$$
r_{i j}=\sum_{k} h_{i k} h_{j k} *
$$

$\delta_{i j}$ is Kroneker's delta, $<>$ is the expectation over the channel matrix. Note that (3) does take into account correlation occurring at both the transmit and receive ends. This equation can be used for statistical (Monte-Carlo) simulations to evaluate $\langle C\rangle$ for some specific models of the channel matrix. However, these matrix numerical computations can be very lengthy, especially when the number of antennas is very large. Here we propose to use Jensen's inequality to obtain an upper bound on $\langle C\rangle$. According to this inequality and concavity of $\log$ det function [9], one obtains:

$$
\langle C\rangle \leq \overline{C_{R}}=\log _{2} \operatorname{det}\left[\delta_{i j}+\frac{\rho}{n} \cdot r_{i j}^{R}\right]
$$

where $r_{i j}^{R}$ is the correlation matrix of receive branches,

$$
r_{i j}^{R}=\sum_{k}\left\langle h_{i k} h_{j k} *\right\rangle,
$$

Note that this correlation matrix does not capture the correlation of transmit branches (since $k$ in (6) represents the transmit antenna index and it is the same for both factors). Thus, the upper limit in (5) can be close to the mean capacity when the correlation of receive branches is much higher than the correlation of transmit branches and, consequently, the effect of transmit branch correlation can be ignored. However, if the transmit correlation is higher than the receive one, then the upper bound in (5) is not an accurate approximation of the mean capacity. Therefore, in order to have an upper bound that is as close as possible to the mean capacity, one must also account for transmit correlation. To this end, the reciprocity of (1) can be used in the following way. First, we note that the MIMO capacity given by (1) is invariant under the transformation $\mathbf{H} \rightarrow \mathbf{H}^{\mathrm{T}}$ (" $\mathrm{T}$ " means transpose). This in effect is equivalent to reversing the direction of information transmission by interchanging transmit and receive ends. Thus, (3) still holds true if we define $r_{i j}$ as:

$$
r_{i j}=\sum_{k} h_{k i} h_{k j}{ }^{*}
$$

Hence, one obtains the second upper bound (the transmit bound),

$$
\langle C\rangle \leq \overline{C_{T}}=\log _{2} \operatorname{det}\left[\delta_{i j}+\frac{\rho}{n} \cdot r_{i j}^{T}\right]
$$

where $r_{i j}^{T}$ is the correlation matrix of transmit branches,

$$
r_{i j}^{T}=\sum_{k}\left\langle h_{k i} h_{k j} *\right\rangle,
$$

Note that the upper bound in (8) does not capture the receive correlation. Therefore, this upper bound will be close to the mean capacity when the transmit correlation is higher than the receive one. However, if the opposite is true, then this upper bound is not an accurate approximation of the mean capacity.

From inequalities (5) and (8) it is clear that a tighter upper bound of the mean channel capacity can be obtained by combining them. Thus, we form the compound upper bound by taking minimum of the two bounds defined above,

$$
\overline{C_{c m p}}=\min \left[\overline{C_{R}}, \overline{C_{T}}\right\rfloor
$$

This upper bound is much tighter than the receive or transmit bound considered separately when the transmit and receive branch correlations are significantly different.

Let us now consider an illustrative example of correlated Rayleigh channel. The components of $\mathbf{H}$ are taken to be identically distributed complex gaussian variables (real and imaginary parts are identically distributed and independent, i.e. the phase is uniformly distributed over $[0,2 \pi]$ ) with zero mean and unit variance. The correlation matrix of $\mathbf{H}$ is assumed to be of the following form:

$$
R_{i j, k m}=\left\langle h_{i k} h_{j m} *\right\rangle=R_{i j}^{R} \cdot R_{k m}^{T},
$$

where $R_{i j}^{R}$ and $R_{i j}^{T}$ are uniform correlation matrixes of the receive and transmit branches correspondingly,

$$
R_{i j}^{R}=\left\{\begin{array}{ll}
r, & i \neq j \\
1, & i=j
\end{array}, \quad R_{k m}^{T}=\left\{\begin{array}{c}
1-r, \quad i \neq j \\
1, \quad i=j
\end{array},\right.\right.
$$

where $0 \leq r \leq 1$. In fact, (11) assumes that the receive and transmit branches are correlated independently on each other (which may be justified by the presence of local scatterers near both ends). Fig. 1 shows the mean capacity of this channel, obtained by extensive numerical simulations (Eq. 3), and the receive (Eq. 5), transmit (Eq. 8) and compound (Eq. 10) bounds. In this example, $r=0$ corresponds to uncorrelated receive branches and full correlation of the transmit ones; $r=1$ corresponds to full correlation of receive branches and uncorrelated transmit ones. The compound bound provides a good approximation to the mean capacity while the receive or transmit bounds alone are not accurate for the whole range of $r$. It is also interesting to note that the maximum capacity is achieved for $r=0.5$. This indicates 


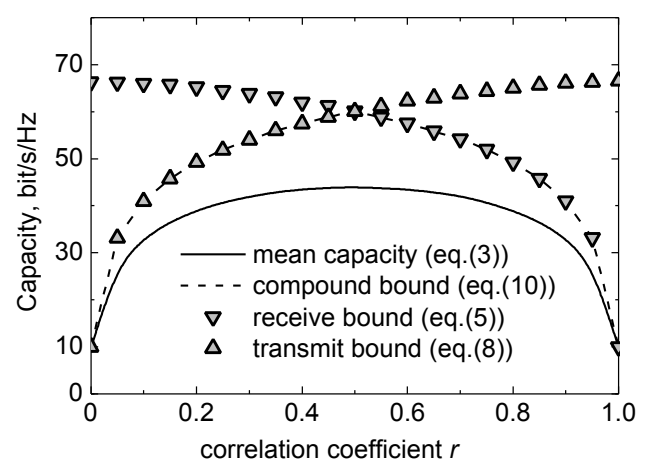

Fig. 1. MIMO channel capacity and its upper bounds versus correlation coefficient

that decrease in capacity is usually due to that side (transmit or receive) which has higher correlation. Thus, a rough estimation of the capacity may be obtained by considering only the higher correlated side.

\section{MIMO CAPACITY IN MULTIPATH ENVIRONMENT}

Following the approach proposed in [12], we employ the spatial correlation matrix model presented in [7] in order to estimate the MIMO capacity in multipath environment. In this model, each user generates many independent multipath signals arriving to the adaptive array within $\pm \Delta$ of the mean angle of arrival (AOA) $\varphi$ (see Fig. 2).

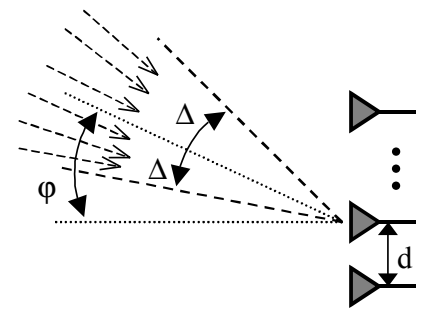

Fig. 2. Incoming multipath signals arrive to the linear antenna array within $\pm \Delta$ of mean angle $\varphi$

The AOA probability density function is assumed to be uniform and all users are assumed to be statistically independent and to have the same statistics. The normalized signal correlation coefficient between the i-th and k-th antenna array elements is:

$$
R_{i k}=\frac{1}{2 \Delta} \int_{\varphi-\Delta}^{\varphi+\Delta} \exp [j z(i-k) \sin \beta] d \beta
$$

where $z=2 \pi d / \lambda, d$ is the inter-element distance, $\lambda$ is the wavelength, $2 \Delta$ is the angle spread of the incoming multipaths, $\varphi$ is the average angle of arrival, and $j$ is the imaginary unit. Without loss of generality, we assume that $\lambda=1$. For $\Delta=\pi$, eq. (13) reduces to the classical expression:

$$
R_{i k}=J_{0}[z(i-k)]
$$

where $\mathrm{J}_{0}$ is the zero-order Bessel function of the first kind. For $\Delta<\pi$ a Bessel series expansion was derived for $R_{i k}$ in [7]. However, as detailed analysis shows, for small $\Delta$ (a few degrees) this expansion converges very slowly and, consequently, a large number of terms must be used in order to estimate $R_{i k}$ accurately. The computational efficiency of this procedure is very low, especially when matrix computations are involved, as is the case for MIMO systems. A simple but still accurate approximation of (13) for small $\Delta$ and $\varphi=0$ can be derived using $\sin \beta \approx \beta$ (valid for small $\beta$ ), and performing integration in (13):

$$
R_{i k} \approx \frac{\sin z(i-k) \Delta}{z(i-k) \Delta}
$$

The smaller $\Delta$, the better the accuracy is. Thus, this approximation works exactly where it is needed. The upper bound of its validity is approximately $\pi / 4$. Hence, one may use (14) for large values of $\Delta$ and (15) for small.

In order to study the effect of correlation in an explicit way and to separate it from the effect of unequal received powers, we further assume that all the received powers are equal, i.e. $\quad \sigma_{i}=\sum_{j}\left\langle\left|h_{i j}\right|^{2}\right\rangle=1$. Then, $r_{i j}$ in (6) is the normalized correlation matrix, i.e. $\left|r_{i j}\right| \leq 1$. Hence, one can apply (13)-(15) to model it in the multipath environment of Fig. 2 and (5) gives an upper bound on the mean capacity of such a channel. As detailed analysis shows, the upper bound estimated in this way is quite close to the mean capacity when all the correlation is due to the receive part of the system (i.e., when $\left\langle h_{i k} h_{i m} *\right\rangle=0$ for $k \neq m$ ) and when the channel is not a degenerate one $[9,10]$. Thus, $\overline{C_{R}}$ may be used as a rough estimation of $\langle C\rangle$ in this case. Note that $\overline{C_{R}}$ is simple to evaluate numerically (for a given $\mathbf{R}$ ) while $\langle C\rangle$ requires lengthy Monte-Carlo simulations. Due to the reciprocity of (1), the effect of transmit branch correlation can be analyzed in a similar way.

In order to estimate the mean capacity by Monte-Carlo simulations, we employ some additional assumptions: (i) there are $N$ multiple paths arriving to each receive antenna from a given transmit antenna, (ii) the angles-of-arrival (AOA) of these paths are uniformly distributed within $\pm \Delta$ of $\varphi$, (iii) the gains of these multiple paths are i.i.d. complex Gaussian variables (i.e., Rayleigh fading) with zero mean and unit variance, (iv) each transmit antenna launches an independent set of $N$ multiple paths (i.e., independent set of AOAs and path gains) with the same statistical characteristics. According to the assumption of independence and of equal statistical characteristics of all the transmit branches, all the terms in (6) are equal. Thus, we use (13)(15) to evaluate $\overline{C_{R}}$ and Monte-Carlo simulations to evaluate $\langle C\rangle$ for different $\Delta, \varphi$ and $d$. First, the case of $\varphi=0$ is 


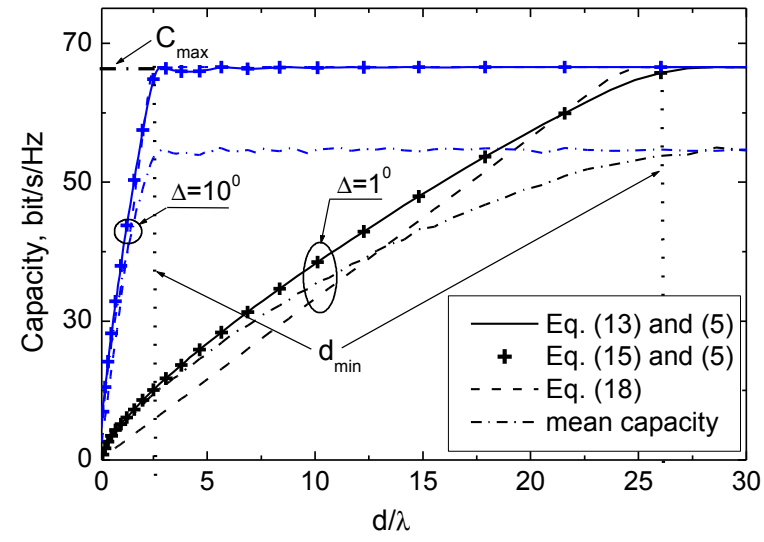

Fig. 3. MIMO capacity of the "average" channel (the upper bound) and the mean (ergodic) capacity versus $d$ for different $\Delta$, $N=20, n=10$ and $\rho=30 \mathrm{~dB}$.

considered (the capacity is maximum under this condition). Fig. 3 shows $\overline{C_{R}}$ and $\langle C\rangle$ versus $d$ for different $\Delta$. Note that there is good agreement between (13) and (15) when estimating the MIMO capacity. Fig. 3 indicates that the function $\overline{C_{R}}(d)$ consists of two regions: (1) for small $d$ $\left(d<d_{\text {min }}\right) \overline{C_{R}}$ increases almost linearly as $d$ increases, (2) for larger $d\left(d>d_{\min }\right) \quad \overline{C_{R}} \quad$ saturates and does not change significantly with $d$. Detailed analysis shows that $d_{\min }$ corresponds approximately to the first zero of $R_{i k}(d)$ for $i=k \pm 1$. Using (15), we obtain:

$$
d_{\min }=\frac{1}{2 \Delta}
$$

$C_{\max }$ is the channel capacity of an uncorrelated matrix channel,

$$
C_{\max }=n \cdot \log _{2}\left(1+\frac{\rho}{n}\right)
$$

Further we observe that $\overline{C_{R}}(d=0)=C_{0}$ where $C_{0}=\log _{2}(1+\rho)$ is the single-input single-output channel capacity (with the same total radiated power). Thus, $\overline{C_{R}}(d)$ can be approximated by the following piecewise-linear function:

$$
\overline{C_{R}}(d) \approx \min \left\{\frac{d}{d_{\min }} C_{\max }+C_{0}, C_{\max }\right\}
$$

Fig. 3 shows that (18) provides quite a good approximation when $C_{\max }>>C_{0}$. For $\Delta>\pi / 4$ the accuracy of (15) and, consequently, of (16) degrades. In this case the following estimation is more accurate: $d_{\min } \approx 0.5$ which approximately corresponds to the first zero of (14). Thus, in a general case one may use the following estimation: $d_{\min }=\max [1 /(2 \Delta), 0.5]$. It should be noted that the mean capacity follows the same dependence on $d$ as the upper bound except that it is $15 \%$ lower in the saturation region.
Thus, the upper bound provides quite an accurate estimation of the mean capacity when the effect of correlation is substantial, i.e. when $d<d_{\min }$. When $N$ increases, the maximum value of the mean capacity increases too, but by a small amount only. When $N<n$ and each transmit antenna generates the same set of AOAs, the mean capacity reduces substantially, which is in good agreement with [11]. The general dependence of $\langle C\rangle$ on $d$ shown in Fig. 3 is quite stable with respect to the assumptions (i)-(iv) above. For example, if the path gains are assumed to be of equal magnitudes and of independent uniformly distributed phases, the maximum capacity is less than $10 \%$ lower than that shown in Fig. 3. The same is true when each transmit antenna generates the same set of AOAs $(N \geq n)$ and the path gains are i.i.d. complex Gaussians. Of course, the assumption of full transmit branch correlation will result in a substantial capacity decrease (see (10)).

Let us now consider the case of $\varphi \neq 0$. Using an analogy with the phased array theory, one may guess that (16) should be generalized to

$$
d_{\min }=\frac{1}{2 \Delta \cos \varphi}
$$

Detailed analysis using extensive numerical simulations shows that this equation is indeed accurate provided that two constraints are satisfied:

$$
\varphi<\pi / 2, \quad \Delta+\varphi \leq \pi / 2
$$

A general form of the function $\overline{C_{R}}(d)$ in this case is the same as in (18). It should be noted that the results of this section can be obtained using the recent results of eigenvalue analysis of diversity combining [8].

\section{TRADEOFF BETWEEN CAPACITY AND DIVERSITY ORDER}

In the discussion so far we considered the MIMO channel capacity. However, this architecture can also provide substantial reduction in fade depth, like conventional diversity combining systems. In this respect, diversity order is an important parameter. Unfortunately, it is not possible to achieve the maximum capacity and diversity order at the same time. The recently-proposed space-time codes [14] may achieve diversity order $n^{2}$ only at the expense of low capacity (i.e., much lower than in (17)). Here we give a simple explanation to this tradeoff, which is general enough to cover any space-time code. In fact, this tradeoff is a feature of the MIMO architecture itself regardless of which space-time code is implemented in the architecture.

Let us consider the MIMO architecture shown on Fig. 4.

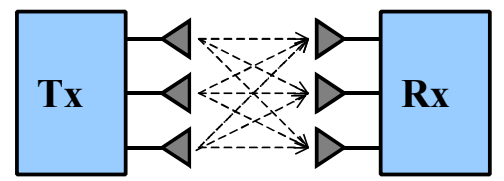

Fig. 4. High-level MIMO architecture, $n=3$. 
In order to achieve the maximum diversity order $\left(n^{2}\right)$, each Tx antenna must launch the same information bits (however, not necessarily at the same time) simply because diversity means that the bit-bearing signal travels along many paths and its copies are combined at the receiver. Thus, diversity order $n^{2}$ means that the signal travels along $n^{2}$ different paths, which is possible only when each Tx antenna launches the same information bits (possibly at different time instants). Note that the total number of paths in Fig. 4 is $n^{2}$ (by "path" we mean a link between each Tx and each Rx antenna, i.e. we do not count multipath components). On the other hand, in order to achieve the maximum capacity, each Tx antenna must launch an independent bit stream [2]. Hence, the maximum diversity order $n^{2}$ is not possible in this mode. In fact, a diversity order of at most $n$ is only possible simply because each bit-bearing signal travels along only $n$ different paths. Note also that the $\mathrm{Rx}$ signal-processing algorithm does not allow to achieve even this diversity order for every bit [2]. From the argument above, we conclude that the maximum diversity order $n_{\mathrm{D}}$ and the maximum channel number $n_{\mathrm{C}}$ (channel number is the number of virtual parallel channels created by the Rx signal processing) are related as:

$$
n_{D} n_{C} \leq n m
$$

where $n$ is the number of Rx antennas and $m$ is the number of Tx antennas $(n \geq m)$. Fig. 5 illustrates this tradeoff. Any

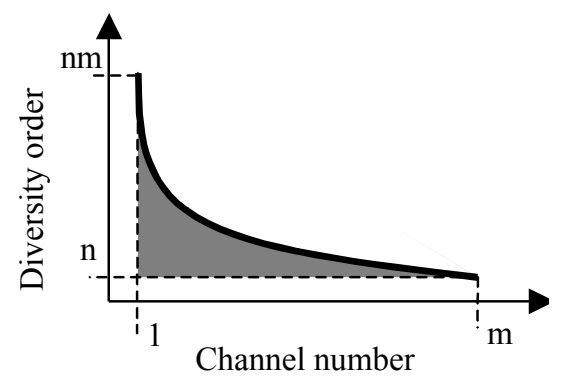

Fig. 5. Channel number-diversity order diagram

actual diversity order and channel number created by using a space-time code must be within the shadowed area. In fact, (21) constitutes a fundamental tradeoff in the space-time code performance since $n_{\mathrm{C}}$ may be roughly viewed as a factor in front of the $\log$ in (17) (it is very similar to the capacity slope [11], the number of effective degrees of freedom [3] and the effective dimensionality [10]). Thus, the channel number limitation transforms to the capacity limitation and, consequently, to the bit rate limitation. For example, for the maximum diversity order $n_{D}=n m, n_{C}=1$ and, consequently, the MIMO capacity is low, i.e. the same as SISO capacity.

\section{CONCLUSIONS}

A new compound upper bound on the mean (ergodic) MIMO channel capacity, which accounts for both transmit and receive branch correlation in such a way that their impact can be estimated separately and a conclusion can be made as to which site contributes more to capacity reduction (which is not easy to do using the mean or outage capacity), has been derived in this paper using the correlation matrix approach. The compound upper bound is tighter than the Tx or $\mathrm{Rx}$ bounds alone and it is not limited to some particular scenarios. Using the bound above, we estimated the MIMO capacity in a correlated multipath environment and demonstrated that the impact of channel correlation on the MIMO capacity is negligible when the two-element array beamwidth is smaller than the angular spread of the incoming multipath signals, which agrees well with the results in [7].

A fundamental tradeoff between the MIMO capacity and diversity order has also been discussed. This tradeoff limits achievable capacity (bit rate) for a given diversity order (or vise versa) for any space-time code.

\section{ACKNOWLEDGEMENTS}

The authors wish to thank F. Gagnon, N. Batani, J. Belzile and $\mathrm{G}$. Tsoulos for useful discussions.

\section{REFERENCES}

[1] G.J. Foschini, M.J Gans, 'On Limits of Wireless Communications in a Fading Environment when Using Multiple Antennas', Wireless Personal Communications, vol. 6, No. 3, pp. 311-335, March 1998.

[2] G.D. Golden et al, 'Detection Algorithm and Initial Laboratory Results Using V-BLAST Space-Time Communication Architecture', Electronics Letters, vol. 35, No. 1, pp.14-16, $7^{\text {th }}$ January 1999.

[3] D.S. Shiu et al, 'Fading Correlation and Its Effect on the Capacity of Multielement Antenna Systems,' IEEE Trans. on Communications, v. 48, N. 3, Mar. 2000, pp. 502-513.

[4] S.L. Loyka, 'Channel Capacity of Two-Antenna BLAST Architecture,' Electronics Letters, vol. 35, No. 17, pp. 1421-1422, 19th Aug. 1999.

[5] S.L. Loyka, J.R. Mosig, 'Channel Capacity of N-Antenna BLAST Architecture,' Electronics Letters, vol. 36, No.7, pp. 660-661, Mar. 2000.

[6] C.C. Martin, J.H. Winters, N.S. Sollenberger, Multiple-Input MultipleOutput (MIMO) Radio Channel Measurements, IEEE VTC'2000 Fall Conference, Sept. 24-28 2000, Boston, USA.

[7] J. Salz, J.H. Winters, Effect of Fading Correlation on Adaptive Arrays in Digital Mobile Radio, IEEE Trans. Vehicular Technology, vol. 43, N. 4, pp. 1049-1057, Nov. 1994.

[8] T. Inoue, Y. Karasawa, Theoretical Analysis on the Performance of Optimal Combining for Multipath Waves Distributed in Spatial and Time Domains, IEICE Trans. Commun., vol. E83-B, N. 7, Jul. 2000, pp. 1426-1434

[9] S. Loyka, A. Kouki, On the Use of Jensen's Inequality for MIMO Channel Capacity Estimation, Canadian Conference on Electrical and Computer Engineering (CCECE 2001), May 13-16, Toronto, Canada, 2001.

[10] S. Loyka, A. Kouki, Correlation and MIMO Communication Architecture (Invited), 8th International Symposium on Microwave and Optical Technology, Montreal, Canada, June 19-23, 2001.

[11] G.G. Rayleigh, J.M. Gioffi, "Spatio-Temporal Coding for Wireless Communications," IEEE Trans. Commun., v.44, N.3, pp. 357-366, 1998.

[12] S. Loyka, G. Tsoulos, Estimating MIMO System Performance Using the Correlation Matrix Approach, IEEE Communication Letters, 2001, submitted.

[13] I.E. Telatar, "Capacity of Multi-Antenna Gaussian Channels," AT\&T Bell Lab. Internal Tech. Memo., June 1995 (European Trans. Telecom., v.10, N.6, Dec.1999).

[14] V. Tarokh, N. Seshadri, A.R. Calderbank, Space-Time Codes for High Data Rate Wireless Communication: Performance Criterion and Code Construction, IEEE Trans. Information Theory, v. 44, N. 2, pp. 744765, Mar. 1998. 